\title{
Asymptotics of entire functions and a problem of hayman
}

Article

Accepted Version

Hilberdink, T. (2020) Asymptotics of entire functions and a problem of hayman. Quarterly Journal of Mathematics, 71 (2). pp. 667-676. ISSN 0033-5606 doi:

https://doi.org/10.1093/qmathj/haz061 Available at https://centaur.reading.ac.uk/88558/

It is advisable to refer to the publisher's version if you intend to cite from the work. See Guidance on citing.

To link to this article DOI: http://dx.doi.org/10.1093/qmathj/haz061

Publisher: Oxford University Press

All outputs in CentAUR are protected by Intellectual Property Rights law, including copyright law. Copyright and IPR is retained by the creators or other copyright holders. Terms and conditions for use of this material are defined in the End User Agreement.

\section{www.reading.ac.uk/centaur}

\section{CentAUR}

Central Archive at the University of Reading

Reading's research outputs online 


\title{
Asymptotics of entire functions and a problem of Hayman ${ }^{1}$
}

\author{
Titus Hilberdink \\ Department of Mathematics, University of Reading, Whiteknights, \\ PO Box 220, Reading RG6 6AX, UK; t.w.hilberdink@reading.ac.uk
}

\begin{abstract}
In this paper we study entire functions whose maximum on a disc of radius $r$ grows like $e^{h(\log r)}$ for some function $h(\cdot)$. We show that this is impossible if $h^{\prime \prime}(r)$ tends to a limit as $r \rightarrow \infty$, thereby solving a problem of Hayman from 1966. On the other hand we show that entire functions can, under some mild smoothness conditions, grow like $e^{h(\log r)}$ if $h^{\prime \prime}(r) \rightarrow \infty$.
\end{abstract}

2010 AMS Mathematics Subject Classification: 26A12, 30D15

Keywords and phrases: entire functions, orders of growth, asymptotic behaviour.

\section{$\S 1$. Introduction}

It is well known that entire functions cannot exhibit any possible growth rate at infinity. For example, if $f$ is entire and $f(z) \ll|z|^{A}$ for $|z|>1$ for some $A>0$, then $f$ must be a polynomial. Thus $|f(z)| \sim c|z|^{\alpha}$ as $|z| \rightarrow \infty$ is impossible for $\alpha$ not an integer, as is say, $|f(z)| \sim|z| \log |z|$. Perhaps less well known is the fact that if $g$ is a sufficiently smooth function growing faster than any polynomial but slower than $e^{\varepsilon(\log x)^{2}}$ for every $\varepsilon>0$, then there is no entire function $f$ with

$$
M(x):=\max _{|z|=x}|f(z)| \sim g(x)
$$

$(\text { see [7] })^{2}$. Indeed, they show that even a growth rate of $g(x)^{1+\varepsilon(x)}$ is impossible for functions $\varepsilon(x)$ tending to zero at a certain rate. For example,

$$
M(x)=e^{c(\log x)^{\lambda}+o\left((\log x)^{2-\lambda}\right)}
$$

is impossible for $1<\lambda<2$ and $c>0$. On the other hand, they show that if " $O$ " is replaced by " $O$ ", then it is possible to find an entire function with such growth. For larger functions, it is known that for sufficiently nice $g(x) \geq e^{c(\log x)^{2}}$ for some $c>0$, it is possible to find entire $f$ such that $M(x) \asymp g(x)$ (see [3]). Whether one can obtain $M(x) \sim g(x)$ was left open. (See also [8], where the authors are asking for $M(x) \asymp V(x)$ for some prescribed $V(x)$ rather than $M(x) \sim V(x)$.) That we need $g$ sufficiently nice for (1.1) to hold is clear; for example, if

$$
f(z)=\sum_{n=0}^{\infty} a_{n} z^{n}
$$

with coefficients $a_{n}$ all real and non-negative, then $f$ is infinitely differentiable and each of its derivatives in increasing. So, for example, we cannot have $g(x)=e^{x+2 \sin x}$. For if

$$
f(x) \sim e^{x+2 \sin x},
$$

\footnotetext{
${ }^{1}$ To appear in the Quarterly Journal of Mathematics

${ }^{2}$ The symbols $\sim$ and $\asymp$ are defined as usual: $f(x) \sim g(x)$ if $\lim _{x \rightarrow \infty} \frac{f(x)}{g(x)}=1$ and $f(x) \asymp g(x)$ if there exist $a, b>0$ such that $a \leq \frac{f(x)}{g(x)} \leq b$ for all $x$ sufficiently large.
} 
then for $n \in \mathbb{N}$,

$$
1 \geq \frac{f\left(2 n \pi+\frac{\pi}{2}\right)}{f(2 n \pi+\pi)} \sim \frac{e^{2 n \pi+\frac{\pi}{2}+2}}{e^{2 n \pi+\pi}}=e^{2-\frac{\pi}{2}}
$$

as $n \rightarrow \infty-$ a contradiction.

The peculiar phenomenon that occurs around $e^{c(\log x)^{2}}$ can be seen when we take $a_{n}=e^{-\alpha n^{2}}$ :

$$
\sum_{n=0}^{\infty} e^{-\alpha n^{2}} x^{n}=\sqrt{\frac{\pi}{\alpha}} e^{\frac{1}{4 \alpha}(\log x)^{2}}\left(1+2 \sum_{n=1}^{\infty} e^{-\frac{\pi^{2} n^{2}}{\alpha^{2}}} \cos \left(\frac{\pi n \log x}{\alpha}\right)\right)+O\left(\frac{1}{x}\right) .
$$

(This easily follows from the identity $\sum_{n \in \mathbb{Z}} e^{-\alpha n^{2}+\beta n}=\sqrt{\frac{\pi}{\alpha}} e^{\frac{\beta^{2}}{4 \alpha}} \sum_{n \in \mathbb{Z}} e^{-\frac{\pi^{2} n^{2}}{\alpha}-\frac{i \pi \beta n}{\alpha}}$.) In particular, the series on the left is not asymptotically equal to $\lambda e^{\frac{1}{4 \alpha}(\log x)^{2}}$ for any $\lambda$, even though it is $\asymp e^{\frac{1}{4 \alpha}(\log x)^{2}}$. This suggests what we ask for is impossible, at least for $g(x)=e^{c(\log x)^{2}}$.

\section{A problem of Hayman}

With $f$ and $M$ as above, let $b(r)$ denote the function

$$
b(r)=\left(r \frac{d}{d r}\right)^{2} \log M(r)=\left(\frac{d}{d \log r}\right)^{2} \log M(r) .
$$

Alternatively, writing $M\left(e^{x}\right)=e^{K(x)}$, we have $b(x)=K^{\prime \prime}(x)$. We note that $b(r)$ exists for all $r$ except possibly at isolated points, and that $b(r)$ is continuous away from these points. In any case, $b(r \pm 0)$ does exist and these are both non-negative - a result that follows from Hadamard convexity. Much research has been devoted to studying this function (see for example [1], [4], $[6])$.

The results in [7] were in part inspired by a paper of Hayman [4] where he showed that if $f$ is transcendental entire, then $\limsup _{r \rightarrow \infty} b(r) \geq A_{0}$, some absolute constant and $A_{0} \geq 0.18$. He ended his paper by asking if it is possible to have $b(r) \rightarrow c$ as $r \rightarrow \infty$ for some $c \geq 0$ (indeed one must have $\left.c \geq A_{0}\right)$. Note that, implicitly, this assumes $b(r)$ exists for all $r$ sufficiently large. This natural question appears never to have been answered. With the help of Theorem 1 below, we now have a solution.

\section{Theorem 1}

Let $k:(a, \infty) \rightarrow \mathbb{R}$ be twice continuously differentiable and such that (i) $k^{\prime \prime}(x)>0$ for all $x \geq a$ and $k^{\prime \prime}(x) \rightarrow c$ for some $c \geq 0$, and (ii) $k^{\prime}(x) \rightarrow \infty$ as $x \rightarrow \infty$. Then there is no entire function $f$ for which

$$
M(r) \sim e^{k(\log r)} \quad \text { as } r \rightarrow \infty .
$$

Corollary 2 (Hayman's problem [4])

Let $f(z)$ be entire and transcendental with $M(r)$ and $b(r)$ as before. Then $\lim _{r \rightarrow \infty} b(r)$ does not exist.

Proof. Suppose the limit does exist and equals, say, $c$. Define $k(x)$ via $k^{\prime \prime}(x)=b(x)$ (exists and is continuous for all $x$ large. By extending $k$ to $[0, \infty)$ if necessary, we see that $k$ satisfies the conditions of Theorem 1. But then $M\left(e^{x}\right) \sim e^{k(x)}$, and we have a contradiction. 
On the other hand, for larger $g$, we can find entire functions satisfying (1.1). For this we shall require the notion of regularly varying functions (see [2]). A measurable function $\phi:(a, \infty) \rightarrow$ $(0, \infty)$ is regularly varying of index $\rho$ if

$$
\phi(\lambda x) \sim \lambda^{\rho} \phi(x) \quad \text { as } x \rightarrow \infty \text { for all } \lambda>0 .
$$

Note that, as such, $\phi(x)=x^{\rho+o(1)}$ and $\phi(x+o(x)) \sim \phi(x)$.

First we prove the following result, which is closely reminiscent of a result of Hayman [5] giving the asymptotic behaviour of the coefficients of a power series. Here though, we obtain the asymptotic behaviour of the function given information of the coefficients rather than the other way round.

\section{Theorem 3}

Let $k:[0, \infty) \rightarrow \mathbb{R}$ be twice continuously differentiable and such that $k, k^{\prime}, k^{\prime \prime}>0$ on $(0, \infty)$ with $k(0)=k^{\prime}(0)=0$ and $k^{\prime \prime}(x) \rightarrow \infty$ as $x \rightarrow \infty$. Let $\ell=\left(k^{\prime}\right)^{-1}$ and suppose that $\ell^{\prime}$ is regularly varying of index $-\alpha$ with $\alpha \in[0,1]$. Put $L(x)=\int_{0}^{x} \ell$. Then

$$
\sum_{n=0}^{\infty} e^{-L(n)+n y} \sim \sqrt{2 \pi k^{\prime \prime}(y)} e^{k(y)} \quad \text { as } y \rightarrow \infty .
$$

Based on this, we obtain:

\section{Theorem 4}

Let $h:(a, \infty) \rightarrow \mathbb{R}$ be a $C^{4}$-function such that $h^{\prime \prime}(x) \rightarrow \infty$ as $x \rightarrow \infty$, and

$$
h^{\prime \prime \prime}=o\left(\left(h^{\prime \prime}\right)^{3 / 2}\right), h^{(4)}=o\left(\left(h^{\prime \prime}\right)^{2}\right) .
$$

Further assume that $m:=\left(h^{\prime}\right)^{-1}$ is such that $m^{\prime}$ is regularly varying of index $-\alpha$ with $\alpha \in[0,1]$. Then there is a sequence $\left(a_{n}\right)_{n \geq 0}$ with $a_{n} \geq 0$ for which

$$
\sum_{n=0}^{\infty} a_{n} x^{n} \sim e^{h(\log x)} \quad \text { as } x \rightarrow \infty .
$$

Remark. The conditions in (1.3) do not seriously restrict the size of $h$. Typically for large (nice) functions $F$ one has $F^{\prime}=(F)^{1+o(1)}$, so one would expect $h^{\prime \prime \prime}, h^{(4)}=\left(h^{\prime}\right)^{1+o(1)}$ and $(1.3)$ holds. For small (nice) $h$ (with $h^{\prime \prime} \rightarrow \infty$ ) we typically expect $h^{\prime \prime \prime}, h^{(4)}=o\left(h^{\prime}\right)$ so (1.3) holds again.

\section{$\S 2$. Proofs}

The main result (Theorem 1) concerns $c>0$ but we can deal with $c=0$ at the same time, so we include it. Note that this case is much simpler. Essentially, we prove (1.2) is also impossible for $\lambda=2$. As noted, this case was excluded from [7] and indeed our methods are rather different, using a sequence of nested sequences.

Proof of Theorem 1. Without loss of generality we may extend $k$ to a $C^{2}$-function defined on $[0, \infty)$ such that $k^{\prime}$ is strictly increasing. Suppose we can find an entire function

$$
f(z)=\sum_{n=0}^{\infty} a_{n} z^{n}
$$


for which $M(r) \sim e^{k(\log r)}$ as $r \rightarrow \infty$. By Cauchy's inequality, we have

$$
\left|a_{n}\right| \leq M\left(e^{y}\right) e^{-n y} \sim e^{k(y)-n y}
$$

for all $y \geq 0$. The optimal value is to choose $y$ such that $k^{\prime}(y)=n$. This is uniquely given, say $y=y_{n}$, as $k^{\prime}$ is strictly increasing and continuous. Hence, we may write

$$
a_{n}=b_{n} e^{k\left(y_{n}\right)-n y_{n}} \quad \text { where }\left|b_{n}\right| \lesssim 1 .
$$

Now the assumption on $M$ implies that

$$
\sum_{n=0}^{\infty} b_{n} e^{-w_{n}(y)} \rightarrow 1 \quad \text { as } y \rightarrow \infty
$$

where

$$
w_{n}(y)=k(y)-k\left(y_{n}\right)-n\left(y-y_{n}\right)=\int_{y_{n}}^{y} k^{\prime}(t)-n d t=\int_{y_{n}}^{y} \int_{y_{n}}^{t} k^{\prime \prime}(u) d u d t .
$$

Since $k^{\prime \prime}>0$, we have $w_{n}(y) \geq 0$ with equality if and only if $y_{n}=y$; i.e. if and only if $n=k^{\prime}(y)$.

Define $\ell$ (on a neighbourhood of infinity) to be the inverse of $k^{\prime}$; i.e. $k^{\prime}(\ell(x))=x$ and $y_{n}=\ell(n)$. By differentiating we see that, as $x \rightarrow \infty$,

$$
\ell^{\prime}(x) \rightarrow\left\{\begin{array}{cc}
\frac{1}{c} & \text { if } c>0 \\
\infty & \text { if } c=0
\end{array} .\right.
$$

From (2.2) we have, with $t=\ell(v)$

$$
w_{n}(y)=\int_{n}^{k^{\prime}(y)}(v-n) \ell^{\prime}(v) d v
$$

Thus in either case we have $w_{n}(y) \geq a\left(n-k^{\prime}(y)\right)^{2}$ for some $a>0$ for all $n, y$ sufficiently large.

Put $y=\ell(N+\lambda)$ where $N \in \mathbb{N}$ and $\lambda \in[0,1]$. Then the inequality becomes

$$
w_{N+n}(\ell(N+\lambda)) \geq a(n-\lambda)^{2}
$$

for some $a>0$ and all $N$ sufficiently large such that $n \geq-N+n_{0}$, while (2.1) becomes

$$
\sum_{n=-N}^{\infty} b_{N+n} e^{-w_{N+n}(\ell(N+\lambda))} \rightarrow 1 \quad \text { as } N \rightarrow \infty \text {, uniformly for } \lambda \in[0,1] .
$$

Furthermore,

$$
\sum_{-\sqrt{N} \leq n \leq \sqrt{N}} b_{N+n} e^{-w_{N+n}(\ell(N+\lambda))} \rightarrow 1 \quad \text { as } N \rightarrow \infty, \text { uniformly for } \lambda \in[0,1] .
$$

(Indeed, instead of $\sqrt{N}$ we could take any function $\varphi(N)$ tending to infinity with $N$ such that $\varphi(N)=o(N)$.) Also, we shall see shortly that $w_{N+n}(\ell(N+\lambda)) \rightarrow \frac{(n-\lambda)^{2}}{2 c}$ as $N \rightarrow \infty$, in case $c>0$.

The idea is now to construct a sequence $\left(c_{n}\right)_{n \in \mathbb{Z}}$ from the limit points of the $b_{n}$ such that (2.3) is turned into

$$
\sum_{n \in \mathbb{Z}} c_{n} e^{-\frac{(n-\lambda)^{2}}{2 c}}=1 \quad \forall \lambda \in[0,1]
$$


Let $\mathcal{B}$ denote the set of limit points of $\left(b_{n}\right)$. Note that $\mathcal{B}$ is contained in the closed unit disc.

We define the $c_{n}$ inductively, first for $c_{0}$, then $c_{ \pm 1}, c_{ \pm 2}$ etc. as follows. Let $c_{0}$ be any limit point of $\left(b_{n}\right)$, which exists, as it is bounded; i.e. $c_{0} \in \mathcal{B}$. Thus there is a sequence of $N$ s such that $b_{N} \rightarrow c_{0}$. Now suppose we have defined $c_{n}$ for $|n| \leq k$, for some $k \geq 0$ and that

$$
b_{N+n} \rightarrow c_{n} \quad \text { for }|n| \leq k \text { as } N \rightarrow \infty \text { through some sequence. }
$$

We define $c_{ \pm(k+1)}$ as follows. The sequence $b_{N+k+1}$ (with $N$ taking values in the particular subset of $\mathbb{N}$ as above), being bounded, has a convergent subsequence. Call the limit $c_{k+1}$. Since subsequences of a sequence converge to the same limit as the sequence, we now have

$$
b_{N+n} \rightarrow c_{n} \quad \text { for }|n| \leq k \text { and } n=k+1 \text { as } N \rightarrow \infty \text { through some sequence in } \mathbb{N} \text {. }
$$

Now do the same for $b_{N-(k+1)}$ by taking a further subsequence. This defines $c_{n}$ for $|n| \leq k+1$ and, by induction, we obtain a sequence $\left(c_{n}\right)_{n \in \mathbb{Z}}$ in $\mathcal{B}$. More precisely, the above says that, given $\varepsilon>0$ and $k \in \mathbb{N}$, there exists an $N_{k} \in \mathbb{N}$ and $S_{k}$, an unbounded subset of $\mathbb{N}$, such that for all $|n| \leq k$,

$$
\left|b_{N+n}-c_{n}\right|<\varepsilon \quad \text { for } N \in S_{k} \text { with } N \geq N_{k} .
$$

Note that $S_{m} \subset S_{m-1}$ for every $m \in \mathbb{N}$.

Now for the case when $c>0$, so that $\ell^{\prime} \rightarrow 1 / c$, we have, uniformly for $\lambda \in[0,1]$,

$w_{N+n}(\ell(N+\lambda))=\int_{N+n}^{N+\lambda}(v-N-n) \ell^{\prime}(v) d v=\int_{0}^{\lambda-n} t \ell^{\prime}(N+n+t) d t \rightarrow \frac{(n-\lambda)^{2}}{2 c}$ as $N \rightarrow \infty$.

Thus, in this case, given $\varepsilon>0$ and $k \in \mathbb{N}$, there exists a $N_{k} \in \mathbb{N}$ and $S_{k}$, an unbounded subset of $\mathbb{N}$, such that for all $|n| \leq k$ and all $\lambda \in[0,1]$,

$$
\left|b_{N+n} e^{\frac{(n-\lambda)^{2}}{2 c}-w_{N+n}(\ell(N+\lambda))}-c_{n}\right|<\varepsilon \quad \text { for } N \in S_{k} \text { with } N \geq N_{k} .
$$

Now we show that (2.4) holds. We have

$$
\begin{aligned}
\left|\sum_{n \in \mathbb{Z}} c_{n} e^{-\frac{(n-\lambda)^{2}}{2 c}}-1\right| & =\mid \sum_{|n|>k} c_{n} e^{-\frac{(n-\lambda)^{2}}{2 c}}+\sum_{|n| \leq k}\left(c_{n} e^{-\frac{(n-\lambda)^{2}}{2 c}}-b_{N+n} e^{-w_{N+n}(\ell(N+\lambda))}\right) \\
& -\sum_{k<|n| \leq \sqrt{N}} b_{N+n} e^{-w_{N+n}(\ell(N+\lambda))}+\sum_{|n| \leq \sqrt{N}} b_{N+n} e^{-w_{N+n}(\ell(N+\lambda))}-1 \mid \\
\leq & E_{1}+E_{2}+E_{3}+E_{4},
\end{aligned}
$$

where

$$
\begin{aligned}
& E_{1}=\sum_{|n|>k}\left|c_{n}\right| e^{-\frac{(n-\lambda)^{2}}{2 c}}, \quad E_{2}=\sum_{|n| \leq k}\left|b_{N+n} e^{-w_{N+n}(\ell(N+\lambda))}-c_{n} e^{-\frac{(n-\lambda)^{2}}{2 c}}\right| \\
& E_{3}=\sum_{k<|n| \leq \sqrt{N}}\left|b_{N+n}\right| e^{-w_{N+n}(\ell(N+\lambda))}, \quad E_{4}=\left|\sum_{|n| \leq \sqrt{N}} b_{N+n} e^{-w_{N+n}(\ell(N+\lambda))}-1\right| .
\end{aligned}
$$

Let $\varepsilon>0$. Since $b_{n}$ and $c_{n}$ are bounded we have (for some constant $C$ ),

$$
E_{1}, E_{3}<C \sum_{|n|>k} e^{-\frac{(n-\lambda)^{2}}{2 c}} \leq 2 C \sum_{n \geq k} e^{-\frac{n^{2}}{2 c}}<\varepsilon,
$$


for $k \geq k_{0}$ (dependent on $\varepsilon$ only) and all $\lambda \in[0,1]$. Next, there exists $N^{\prime}$ such that for $N \geq N^{\prime}$,

$$
E_{4}<\varepsilon \quad \forall \lambda \in[0,1]
$$

Next, by (2.6) we have for $N \in S_{k}$ with $N \geq N_{k}$,

$$
E_{2} \leq \varepsilon \sum_{|n| \leq k} e^{-\frac{(n-\lambda)^{2}}{2 c}}<2 \varepsilon \sum_{n=0}^{\infty} e^{-\frac{n^{2}}{2 c}}=C^{\prime} \varepsilon
$$

for some constant $C^{\prime}$. Hence for $N \in S_{k_{0}}$ with $N \geq \max \left\{N^{\prime}, N_{k_{0}}\right\}$, we have

$$
\left|\sum_{n \in \mathbb{Z}} c_{n} e^{-\frac{(n-\lambda)^{2}}{2 c}}-1\right| \leq E_{1}+E_{2}+E_{3}+E_{4}<\left(3+C^{\prime}\right) \varepsilon .
$$

This establishes (2.4). But the function

$$
g(z)=\sum_{n \in \mathbb{Z}} c_{n} e^{-\frac{(n-z)^{2}}{2 c}}
$$

is entire, being a locally uniformly convergent series of holomorphic functions on $\mathbb{C}$. As it is 1 on the interval $[0,1]$ it must be identically 1 . Thus for $x$ real

$$
e^{-\frac{c}{2} x^{2}}=e^{-\frac{c}{2} x^{2}} g(c i x)=e^{-\frac{c}{2} x^{2}} \sum_{n \in \mathbb{Z}} c_{n} e^{-\frac{(n-i c x)^{2}}{2 c}}=\sum_{n \in \mathbb{Z}} c_{n} e^{-\frac{n^{2}}{2 c}} e^{n i x} .
$$

The RHS is periodic while the LHS tends to 0 at infinity but is non-zero. This is a contradiction.

It remains to prove the case $c=0$ is also impossible. For this case take $\lambda=\frac{1}{2}$ in (2.3). Now

$$
w_{N+n}\left(\ell\left(N+\frac{1}{2}\right)\right)=\int_{0}^{\frac{1}{2}-n} t \ell^{\prime}(N+n+t) d t \rightarrow \infty,
$$

as $N \rightarrow \infty$ for each $n \in \mathbb{N}$. Thus (2.3) cannot hold and we have a contradiction.

Proof of Theorem 3. Regarding $n$ as a real variable, we have $\frac{\partial}{\partial n}(n y-L(n))=y-\ell(n)=0$ when $\ell(n)=y$; i.e. $n=k^{\prime}(y)$. Thus $e^{n y-L(n)}$ is largest when $n=k^{\prime}(y)$ in which case $e^{n y-L(n)}=$ $e^{y k^{\prime}(y)-L\left(k^{\prime}(y)\right)}$. Note that

$$
\left(y k^{\prime}(y)-L\left(k^{\prime}(y)\right)\right)^{\prime}=k^{\prime}(y)+y k^{\prime \prime}(y)-\ell\left(k^{\prime}(y)\right) k^{\prime \prime}(y)=k^{\prime}(y) .
$$

Thus $y k^{\prime}(y)-L\left(k^{\prime}(y)\right)=k(y)$. We show that the main contribution to the series comes from the range $\left|n-k^{\prime}(y)\right| \ll \sqrt{k^{\prime \prime}(y)}$. First we find the contribution from this range. Let $n=$ $k^{\prime}(y)+t \sqrt{k^{\prime \prime}(y)}$. Then

$$
\begin{aligned}
n y-L(n) & =y k^{\prime}(y)+t y \sqrt{k^{\prime \prime}(y)}-L\left(k^{\prime}(y)+t \sqrt{k^{\prime \prime}(y)}\right) \\
& =k(y)-\int_{0}^{t \sqrt{k^{\prime \prime}(y)}} \ell\left(k^{\prime}(y)+u\right)-\ell\left(k^{\prime}(y)\right) d u
\end{aligned}
$$


using $\ell\left(k^{\prime}(y)\right)=y$. The integral on the right of $(2.7)$ is

$$
\sqrt{k^{\prime \prime}(y)} \int_{0}^{t} \ell\left(k^{\prime}(y)+v \sqrt{k^{\prime \prime}(y)}\right)-\ell\left(k^{\prime}(y)\right) d v=\int_{0}^{t} v \frac{\ell^{\prime}\left(k^{\prime}(y)+w_{v, y} \sqrt{k^{\prime \prime}(y)}\right)}{\ell^{\prime}\left(k^{\prime}(y)\right)} d v
$$

for some $w_{v, y}$ lying between 0 and $t$ by the Mean Value Theorem and using the fact that $\ell^{\prime}\left(k^{\prime}(y)\right) k^{\prime \prime}(y)=1$. Since $\ell^{\prime}$ is monotonic and $\sqrt{k^{\prime \prime}(y)}=o\left(k^{\prime}(y)\right)$, the integrand is asymptotic to $v$ and the integral is $\sim \frac{t^{2}}{2}$. Thus from $(2.7)$ we have (with $n=k^{\prime}(y)+t \sqrt{k^{\prime \prime}(y)}$ )

$$
e^{-L(n)+n y} \sim e^{k(y)-\frac{t^{2}}{2}}
$$

locally uniformly for $t \in \mathbb{R}$. Let $\varepsilon>0$. Write $n=\left[k^{\prime}(y)\right]+m$, where $m \in \mathbb{Z}$ and $[x]$ denotes the integer part of $x$. Then $m=t \sqrt{k^{\prime \prime}(y)}+O(1)$, so that

$$
\sum_{\left|n-k^{\prime}(y)\right| \leq A \sqrt{k^{\prime \prime}(y)}} e^{-L(n)+n y} \sim e^{k(y)} \sum_{|m| \leq A \sqrt{k^{\prime \prime}(y)}} e^{-\frac{(m+O(1))^{2}}{2 k^{\prime \prime}(y)}} \sim e^{k(y)} \sum_{|m| \leq A \sqrt{k^{\prime \prime}(y)}} e^{-\frac{m^{2}}{2 k^{\prime \prime}(y)}},
$$

since $m=o\left(k^{\prime \prime}(y)\right)$. The sum on the right is asymptotic to

$$
\int_{-A \sqrt{k^{\prime \prime}(y)}}^{A \sqrt{k^{\prime \prime}(y)}} e^{-\frac{x^{2}}{2 k^{\prime \prime}(y)}} d x=\sqrt{k^{\prime \prime}(y)} \int_{-A}^{A} e^{-\frac{x^{2}}{2}} d x=\sqrt{2 \pi k^{\prime \prime}(y)}(1-\eta),
$$

where $0<\eta<\varepsilon$ for $A$ sufficiently large.

Next consider $n<k^{\prime}(y)$. Write $t=-T$, where $T>0$ and use $\ell(x)-\ell(x-u)=\int_{x-u}^{x} \ell^{\prime} \geq u \ell^{\prime}(x)$ for $u>0$. We have

$$
\begin{aligned}
\int_{0}^{t \sqrt{k^{\prime \prime}(y)}} \ell\left(k^{\prime}(y)+u\right)-\ell\left(k^{\prime}(y)\right) d u & =\int_{0}^{T \sqrt{k^{\prime \prime}(y)}} \ell\left(k^{\prime}(y)\right)-\ell\left(k^{\prime}(y)-u\right) d u \\
& \geq \ell^{\prime}\left(k^{\prime}(y)\right) \int_{0}^{T \sqrt{k^{\prime \prime}(y)}} u d u=\frac{T^{2}}{2} .
\end{aligned}
$$

Hence (2.7) implies $e^{-L(n)+n y} \leq e^{k(y)} \cdot e^{-\frac{T^{2}}{2}}$. It follows that, for $A>0$, writing $n=\left[k^{\prime}(y)\right]-m$ (so that $m \leq T \sqrt{k^{\prime \prime}(y)}$ )

$$
\begin{aligned}
\sum_{n \leq k^{\prime}(y)-A \sqrt{k^{\prime \prime}(y)}} e^{-L(n)+n y} & \leq e^{k(y)} \sum_{\substack{m \geq A \sqrt{k^{\prime \prime}(y)}\\
}} e^{-\frac{m^{2}}{2 k^{\prime \prime}(y)}} \ll e^{k(y)} \int_{A}^{\infty} e^{-\frac{x^{2}}{2 k^{\prime \prime}(y)}} d x \\
& <\varepsilon e^{k(y) \sqrt{k^{\prime \prime}(y)}}
\end{aligned}
$$

for $A$ sufficiently large.

For $n>k^{\prime}(y)$, use $\ell\left(k^{\prime}(y)+u\right)-\ell\left(k^{\prime}(y)\right) \geq u \ell^{\prime}\left(k^{\prime}(y)+u\right) \geq u \ell^{\prime}(n)$ in (2.7) to get

$$
e^{-L(n)+n y} \leq e^{k(y)} \cdot \exp \left\{-\int_{0}^{n-k^{\prime}(y)} u \ell^{\prime}(n) d u\right\}=e^{k(y)} \cdot \exp \left\{-\frac{\left(n-k^{\prime}(y)\right)^{2} \ell^{\prime}(n)}{2}\right\} .
$$

Thus

$$
\sum_{n \geq k^{\prime}(y)+A \sqrt{k^{\prime \prime}(y)}} e^{-L(n)+n y} \leq e^{k(y)} \sum_{m \geq A \sqrt{k^{\prime \prime}(y)}} e^{-\frac{m^{2} \ell^{\prime}\left(m+k^{\prime}(y)\right)}{2}}
$$


Split the sum into the ranges $\left.A \sqrt{k^{\prime \prime}(y)} \leq m \leq k^{\prime}(y)\right]$ and $m>k^{\prime}(y)$. On the former use $\ell^{\prime}\left(m+k^{\prime}(y)\right) \geq \ell^{\prime}\left(2 k^{\prime}(y)\right) \gg \ell^{\prime}\left(k^{\prime}(y)\right)=1 / k^{\prime \prime}(y)$. On the latter use, $\ell^{\prime}\left(m+k^{\prime}(y)\right) \geq \ell^{\prime}(2 m)$. As such, the sum in (2.10) is at most

$$
\sum_{A \sqrt{k^{\prime \prime}(y)} \leq m \leq k^{\prime}(y)} e^{-\frac{a m^{2}}{k^{\prime \prime}(y)}}+\sum_{m>k^{\prime}(y)} e^{-\frac{m^{2} \ell^{\prime}(2 m)}{2}}<\sqrt{k^{\prime \prime}(y)} \int_{A}^{\infty} e^{-a x^{2}} d x+O(1)<\varepsilon \sqrt{k^{\prime \prime}(y)}
$$

for $A$ sufficiently large (since $\left.\ell^{\prime}(2 m) m^{2} \gg \sqrt{m}\right)$. Combining $(2.8),(2.9)$ and $(2.11)$ gives the result.

Proof of Theorem 4. The idea is to find an appropriate function $k$ which satisfies the conditions of Theorem 3 and is such that

$$
\sqrt{2 \pi k^{\prime \prime}(y)} e^{k(y)} \sim e^{h(y)} \quad \text { as } y \rightarrow \infty .
$$

Then, with $\ell$ and $L$ as defined in Theorem 3, we have

$$
\sum_{n=0}^{\infty} e^{-L(n)+n y} \sim e^{h(y)}
$$

and, with $a_{n}=e^{-L(n)}$, the result follows.

We choose $k$ to be the function

$$
k(y)=h(y)-\log \sqrt{2 \pi h^{\prime \prime}(y)} .
$$

As such $k^{\prime}=h^{\prime}-\frac{h^{\prime \prime \prime}}{2 h^{\prime \prime}}$ and $k^{\prime \prime}=h^{\prime \prime}-\frac{h^{(4)}}{2 h^{\prime \prime}}+\frac{\left(h^{\prime \prime \prime}\right)^{2}}{2\left(h^{\prime \prime}\right)^{2}}$. The conditions on $h$ imply that $k^{\prime \prime} \sim h^{\prime \prime}$. Hence

$$
\sqrt{2 \pi k^{\prime \prime}(y)} e^{k(y)}=\sqrt{2 \pi k^{\prime \prime}(y)} \frac{e^{h(y)}}{\sqrt{2 \pi h^{\prime \prime}(y)}} \sim e^{h(y)} .
$$

Now $h^{\prime \prime}=\left(h^{\prime}\right)^{\alpha+o(1)}$ so $h^{\prime \prime \prime}=o\left(\left(h^{\prime \prime}\right)^{3 / 2}\right)=o\left(h^{\prime \prime} h^{\prime}\right)$. Hence $k^{\prime} \sim h^{\prime}$ also holds. Thus

$$
m^{\prime}\left(h^{\prime}(y)\right)=\frac{1}{h^{\prime \prime}(y)} \sim \frac{1}{k^{\prime \prime}(y)}=\ell^{\prime}\left(k^{\prime}(y)\right) .
$$

As $m^{\prime}\left(h^{\prime}(y)\right) \sim m^{\prime}\left(k^{\prime}(y)\right)$, it follows that $\ell^{\prime}(x) \sim m^{\prime}(x)$ and so $\ell^{\prime}$ is regularly varying of index $-\alpha$. The conditions of Theorem 3 are therefore satisfied, at least for $k, k^{\prime}, k^{\prime \prime}$ on $(a, \infty)$. But we can clearly extend $k$ such that $k, k^{\prime}, k^{\prime \prime}>0$ on $(0, a)$ and $k(0)=k^{\prime}(0)=0$. The result follows.

\section{References}

[1] F. Abi-Khuzam, Hadamard convexity and multiplicity and location of zeros, Trans. Amer. Math. Soc. 347 (1995) 3043-3051.

[2] N. H. Bingham, C. M. Goldie and J. L. Teugels, Regular variation, Cambridge University Press, 1987.

[3] J. Clunie and T. Kövari, On integral functions having prescribed growth II, Canad. J. Math. 20 (1968) 7-20. 
[4] W.K. Hayman, A note on Hadamard's convexity theorem, in: Entire functions and related parts of analysis, Proc. Sympos. Pure Math. AMS, 1968, 210-213.

[5] W.K. Hayman, A generalisation of Stirling's formula, J. Reine Angew. Math. 196 (1956) 67-95.

[6] R.R. London, A note on Hadamard's three circles theorem, Bull. London Math. Soc. 9 (1977) 182185.

[7] I.V. Ostorvskii and A.E. Üreyen, The growth irregularity of slowly growing entire functions, Functional Analysis and its applications 40 (2006) 304-312. [Translated from Funktsional'nyi Analyz i Ego Prilozheniya 40 (2006) 72-82.]

[8] U. Schmid, On the approximation of positive functions by power series II, Jour. of Approximation Theory 92 (1998) 486-501. 